\title{
Optical Modeling of Thin-Film Amorphous Silicon Solar Cells Deposited on Nano-Textured Glass Substrates
}

\author{
Mohammad Ismail Hossain, Wayesh Qarony \\ American International University-Bangladesh, Kemal Ataturk Avenue Banani, Dhaka, Bangladesh
}

Email address:

wayesh@aiub.edu (W. Qarony), m.hossain@aiub.edu (M. I. Hossain)

\section{To cite this article:}

Mohammad Ismail Hossain, Wayesh Qarony. Optical Modeling of Thin-Film Amorphous Silicon Solar Cells Deposited on Nano-Textured Glass Substrates. Journal of Energy and Natural Resources. Vol. 4, No. 5, 2015, pp. 56-61. doi: 10.11648/j.jenr.20150405.11

\begin{abstract}
We have introduced an approach to establish a methodology for 3D optical simulation that allows analyzing optical losses in the individual layers of a thin-film solar cell structure. Using commercial Finite-Difference Time-Domain (FDTD) tool, where Maxwell's Curl equations were rigorously solved for optimizing such cells, a computer modeling has been performed. We have reported the ways to investigate efficient light-trapping schemes by using periodically textured transparent conductive oxide (TCO) in thin-film amorphous silicon solar cells. The optical effects in small area thin film silicon p-i-n solar cells deposited on glass substrates coated with aluminum doped zinc oxide ( $\mathrm{ZnO}: \mathrm{Al}$ ) have been addressed. In order to enhance the efficiency, TCO surface morphology has been analyzed, where pyramidal and parabolic textured surfaces have been used. For these cells, the quantum efficiency, short-circuit current, total reflectance, and all absorption losses have been successfully computed and analyzed. The investigation was carried out based on our proposed model that exhibits maximum current density of $17.32 \mathrm{~mA} / \mathrm{cm}^{2}$ for the absorbing layer thickness of $300 \mathrm{~nm}$.
\end{abstract}

Keywords: FDTD, Light-trapping, Amorphous Silicon Solar Cell, p-i-n Thin Film, Quantum efficiency, Short-Circuit Current, Textured Surface, $\mathrm{ZnO}: \mathrm{Al}$, TCO

\section{Introduction}

The most promising thin-film technology has tremendous potentiality in reducing fabrication cost, robustness as well as excellent ecological balance sheet [1], [2]. Among thin-film solar cells, hydrogenated based amorphous silicon (a-Si:H) solar cells hold the best growing prospects due to its higher absorption coefficient, large open-circuit voltage $\left(\mathrm{V}_{\mathrm{oc}}\right)$, and less material requirements. But, better performances can only be achieved for a few hundreds of nanometers due to the lower carrier diffusion length. With the synthesis of amorphous silicon materials and the implementations of new design and fabrication technologies over time, the amorphous PV efficiency has led to recent and significant improvements. So far the best conversion efficiency can be seen around $10.86 \%$, along with a $16.52 \mathrm{~mA} / \mathrm{cm}^{2}$ of short-circuit current density have been reported for a-Si:H solar cell in 2012 [3]. But, it is necessary to overcome the existing conversion efficiency limitations for the better light trapping or photon management, which reduces the reflection losses, enhances scattering inside the cell, and increases optical path length in thin film solar cells [4]. Different surface texturing techniques, deposited on transparent conductive oxides (TCO) are used for better light trapping in order to maximize the absorption in the thin absorber layer of amorphous thin film solar cells, which increases the short-circuit current of the cells as well. Simulations based researches are always treated as the best approaches to optimize cell's structures as well as to get best performance fitting parameters, since the complexities, expenditures, and processing time are very high in conventional experimental or manufacturing processes [5].

In this manuscript, the optics in thin-film amorphous silicon (a-Si:H) solar cells with a hydrogenated Silicon Oxide ( $\mathrm{SiO}: \mathrm{H})$ p-layer was investigated based on optical simulations.

The investigation was carried out both for pyramidal as well as parabolic surface textured. In order to study the influences of the introduction of pyramidal and parabolic texturing on external quantum efficiency, short-circuit current, and approximated conversion efficiency, results of smooth substrates were used as a reference. All of the simulations were performed for the nano-textured glass substrate. 


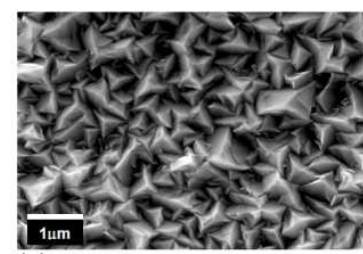

(a)
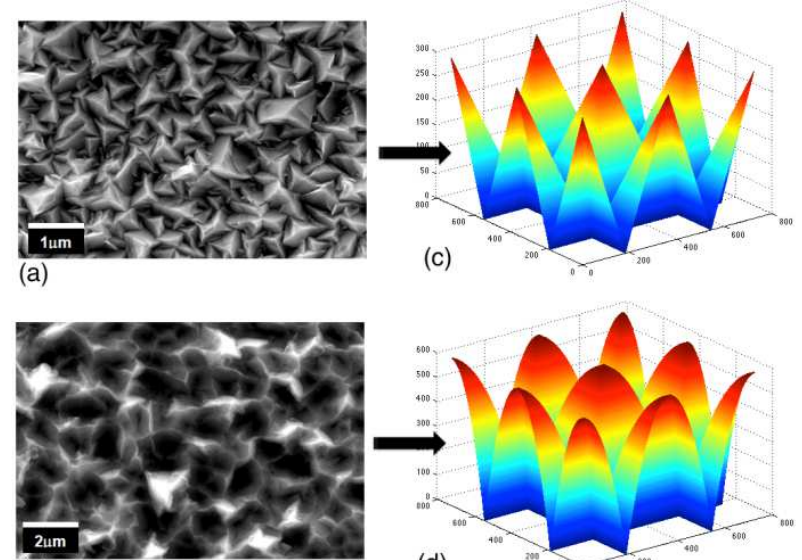

(b) (d)

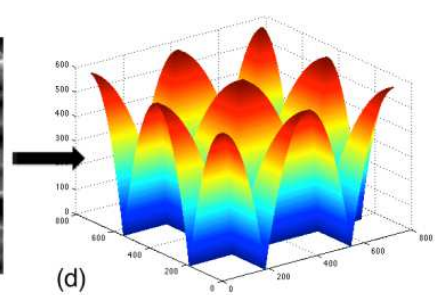

Fig. 1. SEM images of two typical surface morphologies of (a) the standard front LPCVD ZnO optimized for amorphous cells, (b) the new optimized LPCVD ZnO after surface treatment, respectively [taken from 6]; and the corresponding (c) pyramidal and (d) parabolic shapes for the simulations.

\section{Transparent Conductive Oxide Surface}

In order to have an efficient and transparent light trapping in thin-film silicon solar cells, a material with comparatively lower concentration of defects and band-gap above $3.0 \mathrm{eV}$ is required. As the un-doped low-pressure chemical vapor deposition (LPCVD) zinc oxide $(\mathrm{ZnO})$ material has a band-gap of $3.37 \mathrm{eV}$ and the corresponding wavelength is approximately $370 \mathrm{~nm}, \mathrm{ZnO}$ is mostly used as highly transparent conductive oxide (TCO) surface in the visible and near-infrared region [7]. As a consequence, the interface region of transparent conductive oxide absorbs photon energies above band-gap. It also helps to reduce free carrier absorption and increases light-trapping capability, especially for longer wavelengths [6]. Aluminum doped zinc oxide is successfully used in the optical simulations for the front and back contacts to get the efficient light trapping. Depending on the texture surface of TCO, thin-film solar cell efficiency is also realized. From the investigation of experimental surface roughness of TCO, it is possible to get an idea of surface profile dimension. Figure 1(a) and 1(b) show the SEM images of two typical surfaces of chemical vaporization decomposition (CVD) grown of zinc oxide [6]. In order to optimize the solar cell, surface analysis and optical simulations are performed simultaneously. In reality, the surface textures are randomly ordered which is basically the combination of pyramids and asperities can be seen from the Fig. 1(a). By doing the periodic texturing, investigation of optical properties in the solar cells can be achieved.

The correlation between surface roughness and the wave propagation of the optical simulations exhibits the pyramid like textures, where Fig. 1(a) and (c) show the real and simulated surface shapes of textures for this material. In addition, doing surface modification can enhance light trapping or solar cell optimization. In surface morphology treatment, the asperities and the tips of the pyramids are removed and the textured surfaces are turned into mostly parabolic shapes [6]. For the simulation purpose, parabolic shape texturing environment has been created and implemented in our investigation. The experimental optimized pyramidal textured surface and the created parabolic shaped textures for the optical simulations are shown in Fig. 1(b) and $1(\mathrm{~d})$, respectively.

\section{Optical Simulation Model}

A numeric method for computational electromagnetics, the Finite-Difference Time-Domain (FDTD) method has been applied for the optical modeling [8]. The complex refractive index is used to describe the optical properties of the different materials. The excitation source is a monochromatic, harmonic, planar wave with the wavelength that is irradiated perpendicular to the glass/TCO side. The input circular wave propagation or polarization is assumed for the simulation, which is the combination of the transverse electric (TE) and transverse magnetic (TM). At the lateral edges of the structure, periodical boundary conditions are assumed. The simulation domain is closed at top and bottom with a perfectly matched layer (PML). Throughout the simulation, $\mathrm{p}$-i-n single junction thin-film amorphous silicon $(\mathrm{a}-\mathrm{Si}: \mathrm{H})$ solar cell has been investigated. Schematic cross sections of a thin-film amorphous silicon solar cell with smooth substrate and textured interfaces are shown in Fig. 2(a), 3(a), and 4(a).

The amorphous silicon solar cell structure consists of a 420 $\mathrm{nm}$ of $\mathrm{ZnO}$ :Al front contact deposited on a thick $(1000 \mathrm{~nm})$ glass substrate. The zinc oxide layer is followed by a (p-i-n) silicon diode with a specific thickness of cell and a back contact consisting of a $100 \mathrm{~nm}$ of $\mathrm{ZnO}$ :Al layer and a perfect metal back reflector thickness of $1200 \mathrm{~nm}$. The thicknesses of the p-layer and n-layer of the cell have been assumed as $10 \mathrm{~nm}$. Depending on the structure, absorbing layer has been considered for the thickness of $300 \mathrm{~nm}$ and $500 \mathrm{~nm}$. The dimensions of the textured structures are described by the period and height, and the base is assumed to be square shaped.

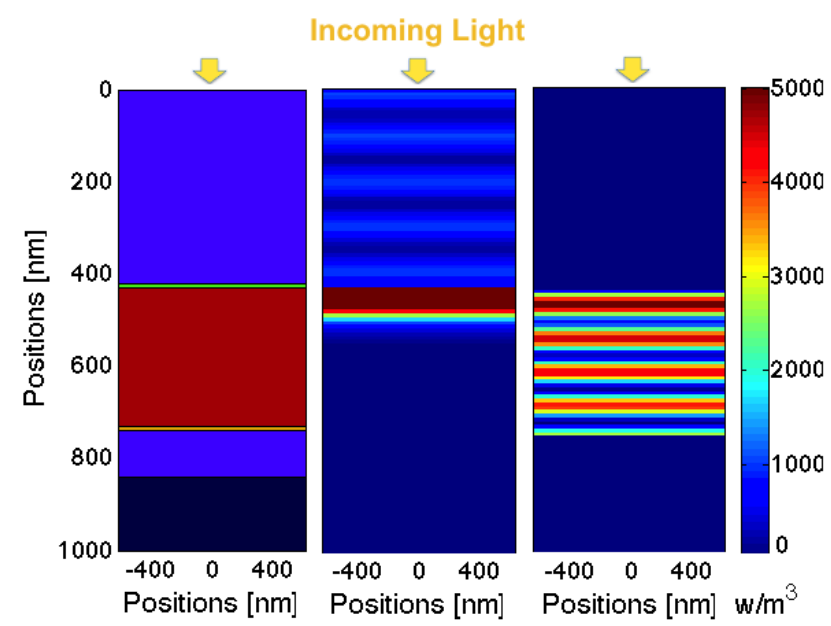

Fig. 2. (a) Schematic of device structure; Simulated power loss profile under monochromatic illumination of wavelength (b) $400 \mathrm{~nm}$ and (c) $600 \mathrm{~nm}$ deposited on smooth glass substrate.

The time average power loss within an amorphous silicon 
solar cell on a smooth substrate is calculated by using equation (1) and shown in Fig. 2(b) and 2(c) for $400 \mathrm{~nm}$ and $600 \mathrm{~nm}$ wavelengths, respectively. For the shorter wavelengths $(<400$ $\mathrm{nm}$ ), photon energy is absorbed within the first few hundreds of nanometers of the solar cell. Due to the high absorption coefficient of amorphous silicon for the shorter wavelength energy, lights cannot reach to the back reflector. The longer wavelengths $(>600 \mathrm{~nm})$ energies propagate through the entire silicon layer and very small amount of lights get reflected from the back. Contrastively, solar irradiance absorption in the solar cell decreases due to further increment of wavelength. Therefore, it is necessary to demonstrate a technique or idea that will enhance the light trapping for the longer wavelength energies as well as effective thickness. In order to increase the effective thickness, there are several techniques demonstrated in several literatures [5]-[6], [8]-[12].

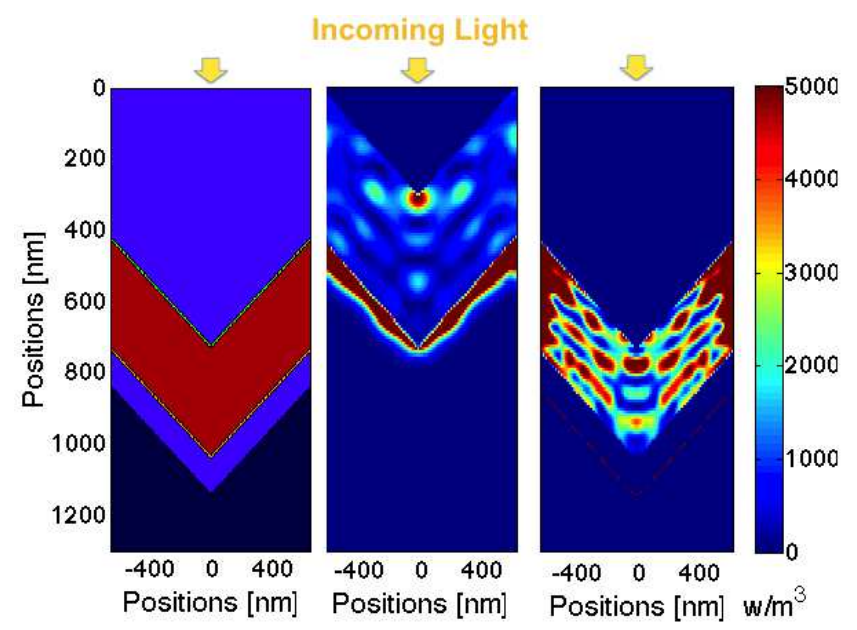

Fig. 3. (a) Schematic of device structure; Simulated power loss profile under monochromatic illumination of wavelength (b) $400 \mathrm{~nm}$ and (c) $600 \mathrm{~nm}$ which deposited on pyramidal textured glass substrate.

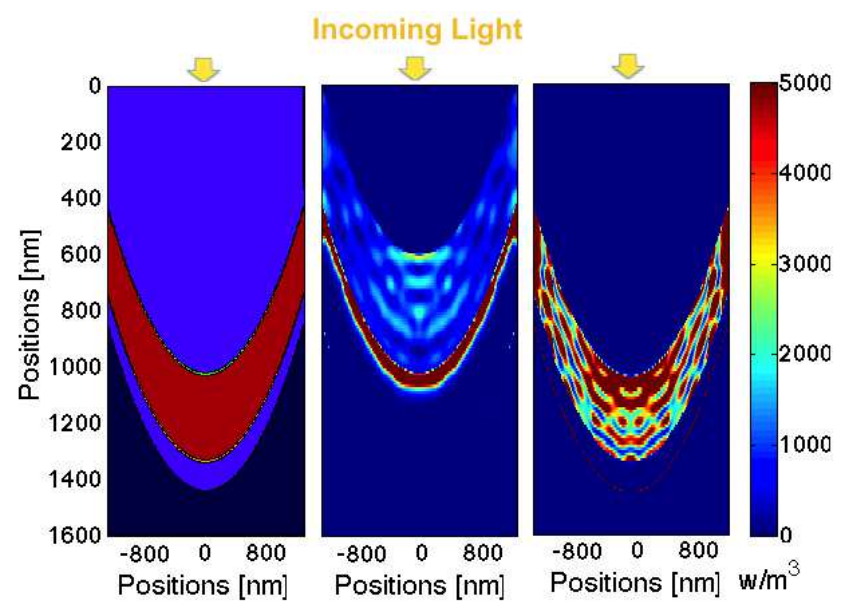

Fig. 4. (a) Schematic of device structure; Simulated power loss profile under monochromatic illumination of wavelength (b) $400 \mathrm{~nm}$ and (c) $600 \mathrm{~nm}$ which deposited on parabolic textured glass substrate.

By introducing the periodic nano-textured interfaces, scattering and diffraction of photons, and the light trapping can be improved. A higher short-circuit current and the efficiency indicators are achieved by textured surface than smooth substrate, since the optical path-length of the cell is increased. The simulated power loss profiles for incident wavelength of $400 \mathrm{~nm}$ and $600 \mathrm{~nm}$ for the textured case are also shown in Figs. 3(b)-3(c) and 4(b)-4(c).

The power loss profile was determined for pyramidal texture with period of $1000 \mathrm{~nm}$ and height of $300 \mathrm{~nm}$, which can be found from the surface analysis of zinc oxide in Fig. 1. In the case of parabolic texture, after surface treatment the period and height are $2000 \mathrm{~nm}$ and $600 \mathrm{~nm}$, respectively. From the electric field distribution, power loss for the individual regions are calculated by the equation no. (1).

$$
Q(x, y, z)=\frac{1}{2} c \varepsilon_{0} n \alpha|E(x, y, z)|^{2}
$$

Where this term leads to find the quantum efficiency, which is expressed by equation no. (2).

$$
Q E=\frac{1}{P_{O p t}} \int Q(x, y, z) d x d y d z
$$

Where $\mathrm{P}_{\text {Opt }}$ is optical power, $\mathrm{c}$ is speed of light in free space, $\varepsilon_{0}$ is permittivity of free space, $\alpha$ is the energy absorption coefficient which is related to extinction coefficient $\mathrm{k}(\alpha=$ $4 \pi \mathrm{k} / \lambda$ ), with $\mathrm{n}$ being the real part of the complex refractive index, and $\mathrm{E}(\mathrm{x}, \mathrm{y}, \mathrm{z})$ is the distributed electric field.

After achieving quantum efficiency and power loss short-circuit current can be calculated by equation no. (3).

$$
I_{S C}=\frac{q}{h c} \int_{\lambda_{\min }}^{\lambda_{\max }} \lambda \cdot E Q(\lambda) \cdot S(\lambda) d \lambda
$$

Here, $h$ is Planck's constant, $\lambda$ is the wavelength, and $S(\lambda)$ is the spectral irradiance of sunlight (AM 1.5). In the above power loss profiles, total wavelength range has been considered from $300 \mathrm{~nm}$ to $800 \mathrm{~nm}$. It was calculated for an incident wave with amplitude of $1 \mathrm{~V} / \mathrm{m}$. For the shorter wavelengths, most of the lights are absorbed within the vicinity of $\mathrm{ZnO}: \mathrm{Al} / \mathrm{Si}$ layer for the $400 \mathrm{~nm}$ wavelength. The power loss in the silicon layer is dominated by the constructive and destructive interferences of the forward and backward propagating waves for the case of $600 \mathrm{~nm}$ wavelength. Due to the low absorption coefficient of silicon in longer wavelengths, a large fraction of the light is reflected from the back contact leading to the formation of a standing wave in front of the back contact. If we compare the field distributions of two textured cases, most of the absorptions are realized in the border of the unit cell in pyramid textured, whereas for the parabolic textured it is done through the bulk of the cell in the longer wavelengths.

\section{Results and Discussions}

In this section, results are shown based on optical wave simulation within thin-film amorphous silicon solar cells for 
wavelengths ranging from $300 \mathrm{~nm}$ to $800 \mathrm{~nm}$. Different structures of the solar cell such as smooth substrate, pyramidal textured, and parabolic textured substrates have been used for the investigation and the schematic cross sections for these cells are shown in Fig. 2(a), 3(a), and 4(a), respectively. At first, the investigations were performed on a smooth substrate used as a reference to compare the performance with textured cells. Of these three structures, solar cell with flat structure was achieved a short-circuit current density of $12.72 \mathrm{~mA} / \mathrm{cm}^{2}$, whereas both pyramidal and parabolic textured cells give approximately same short circuit-current density of 17.32 $\mathrm{mA} / \mathrm{cm}^{2}$. The calculated external quantum efficiency of solar cell with $300 \mathrm{~nm}$ of absorbing layer thickness are shown in Fig 5 , considering $100 \%$ internal quantum efficiency for an incident light spectrum from $300 \mathrm{~nm}$ to $800 \mathrm{~nm}$. All of the three structures exhibit almost similar characteristics for wavelength spectrum shorter than $400 \mathrm{~nm}$, since the incoming lights for shorter wavelength ranges are absorbed in the front of the amorphous silicon solar cell and only a fraction of light can reach in the back contact for diffraction. From the external quantum efficiency (EQE) spectrum of flat solar cell, it is seen that it peaks at almost $82.6 \%$ in the absorption band of around $500 \mathrm{~nm}$, whereas it is about $7.4 \%$ and $6.1 \%$ higher at $600 \mathrm{~nm}$ of optical spectrum for pyramidal and parabolic structures, respectively.

The absorption profiles at these peaks are mainly determined by the interference and superposition of the diffraction modes of the front and back textures. For longer wavelengths, the solar cells with textured surfaces exhibit distinctly enhanced quantum efficiency compared to a solar cell on smooth substrates as the optical path length and effective thickness of the cell are increased by the introduction of the textures.

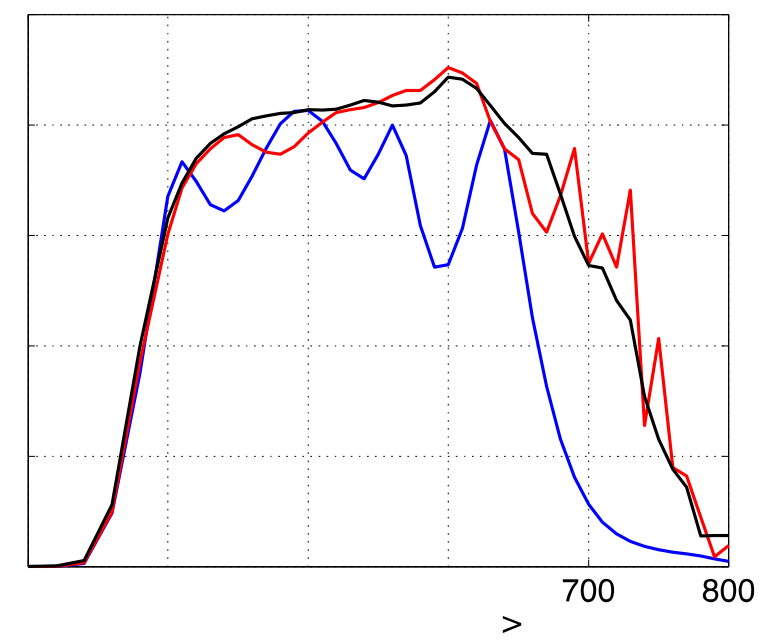

Fig. 5. Comparison of Quantum Efficiencies of $300 \mathrm{~nm}$ intrinsic layer amorphous silicon solar cell for the flat, pyramidal, and parabolic textured of ZnO:Al deposited on glass substrate.

However, a relative decrease in the EQE of all kind of cells is strongly pronounced in the very shorter wavelength (300 $\mathrm{nm}-400 \mathrm{~nm}$ ) due to the parasitic absorption loss in the $\mathrm{ZnO}: \mathrm{Al}$ layer, which is clearly observed in Fig. 7 and 8, the parasitic absorption losses as well as from the power loss profile plotting. Although the external quantum efficiency and absorbance for pyramidal and parabolic textured surfaces are almost same in $300 \mathrm{~nm}$ of absorbing layer cells, distinguishable changes are observed for $500 \mathrm{~nm}$ of photo-active layer depicted in Fig. 6. For blue and infrared part of the spectral wavelength, the absorption for parabolic textured cell is almost always slightly higher than pyramidal textured cell.

As a consequence, the short-circuit current density which is calculated based on external quantum efficiency is 18.48 $\mathrm{mA} / \mathrm{cm}^{2}$ for parabolic textured cell, which is $0.55 \mathrm{~mA} / \mathrm{cm}^{2}$ higher than pyramidal $\left(17.93 \mathrm{~mA} / \mathrm{cm}^{2}\right)$ structure.

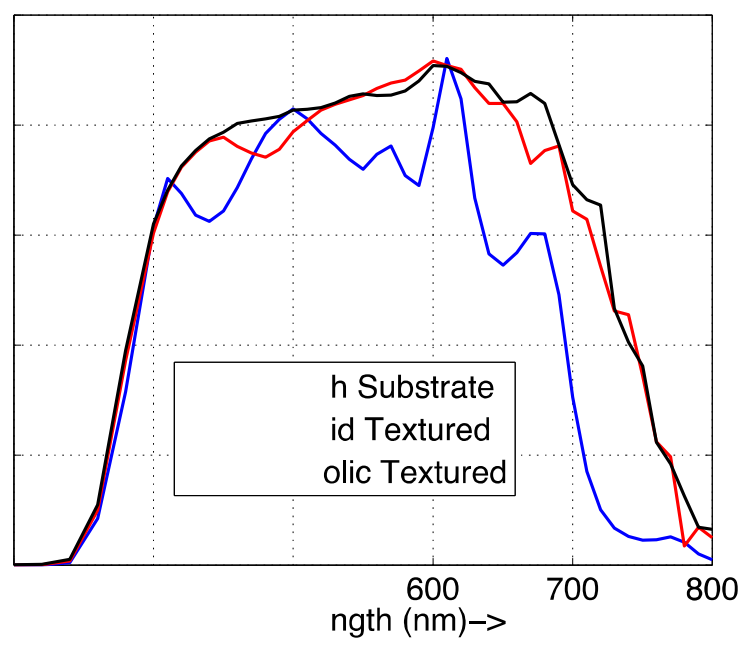

Fig. 6. Comparison of Quantum Efficiencies of $500 \mathrm{~nm}$ intrinsic layer amorphous silicon solar cell for the flat, pyramidal, and parabolic textured of $\mathrm{ZnO}$ :Al deposited on glass substrate.

QE (a-Si:H) $\square$ Reflection $\square$ P doped Si $\square$ N doped Si BTCO-ZnO:Al $\square$ FTCO-ZnO:Al $\square$ Back Reflector-PEC

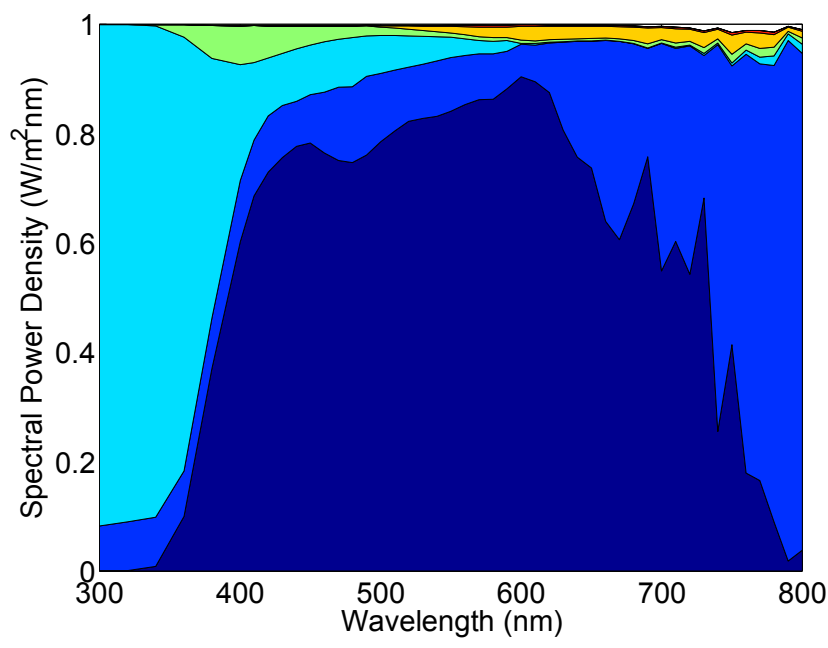

Fig. 7. Layer specific AM1.5G spectral power distribution of a-Si:H pin solar cell with pyramidal textured deposited on glass substrate.

Although the thicker amorphous silicon cell becomes unstable for the realization of open-circuit voltage, an 
investigation was carried out from our own interest to analyze the improvement of the short-circuit current density that is around $1 \mathrm{~mA} / \mathrm{cm}^{2}$.

In order to define the optical losses for all layers in thin-film solar cell, better way to describe with area plot. They are depicted for pyramidal and parabolic in Fig. 7 and 8, respectively, where the influence of two different texturing on the optical properties are visualized.

As the parasitic absorptions in all other layers such as FTCO, p-layer, n-layer, and BTCO do not contribute to the overall short-circuit current as well as the conversion efficiency, these losses have to be minimized in order to boost-up solar cell.
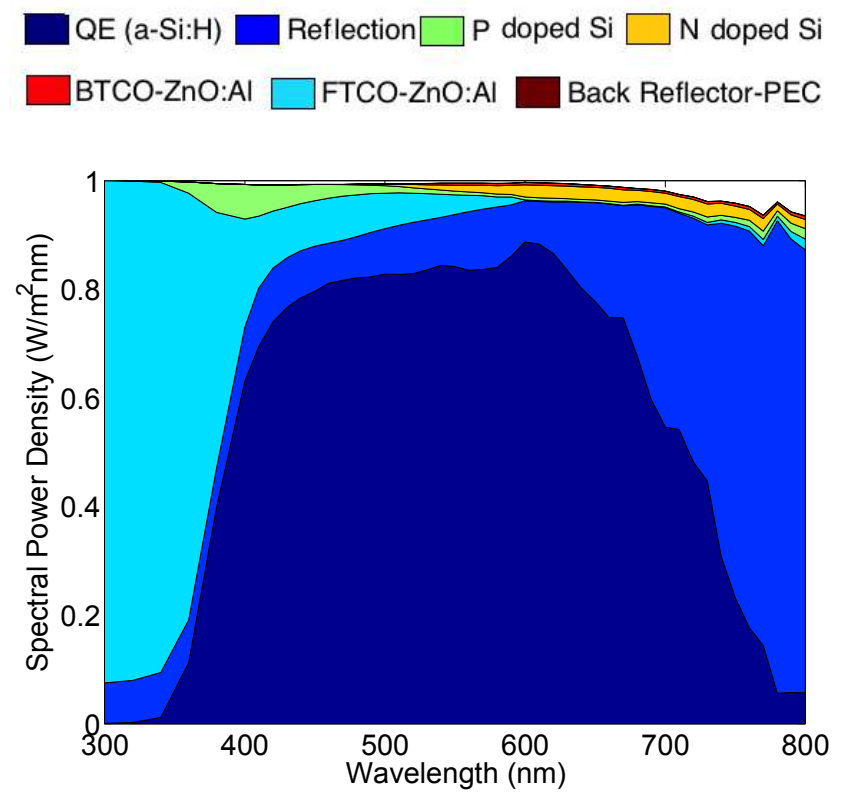

Fig. 8. Layer specific AM1.5G spectral power distribution of a-Si:H pin solar cell with parabolic textured deposited on glass substrate.

In the Fig. 7 and 8, the absorption in the i-layer for the diode is depicted along with parasitic losses in the transparent front TCO, $\mathrm{p}$ and $\mathrm{n}$ doped layers, back contact, and the total reflection. The absorption in the doped p-layer, n-layer, and back contact is very few, which is around $2.7 \%$ of total absorption. Since a perfect electric conductor (PEC) is used as back reflector, the absorption in the back contact is almost zero. The largest loss in the p-layer is observed for the shorter wavelength, whereas it is almost transparent for the longer wavelengths. But, the scenario is just opposite in the case of n-layer. Although the parasitic absorption in the shorter wavelengths is more than $50 \%$ of the total absorption, it is also almost transparent for shorter wavelengths $(<600 \mathrm{~nm})$. It can be observed that for the longer wavelengths, a major amount of incident photon energy is lost as reflection. Our studies on different textured structures have shown that the amount of reflection is decreased in the case of parabolic structure, which enhances the external quantum efficiency. It is also clearly observed that all the grooves at shorter and longer wavelengths are removed and it gets smoother for parabolic surface texturing.

\section{Conclusion}

We have presented optical modeling for the $\mathrm{p}-\mathrm{i}-\mathrm{n}$ single junction thin-film amorphous silicon solar cell in superstrate configuration that is deposited on flat and textured glass substrates coated with transparent conductive oxide (TCO). Fundamental limitations for the single junction thin film amorphous silicon solar cell were discussed in terms of open-circuit voltage and short-circuit current. In addition, for the optimization of cell, we investigated the real TCO surface morphologies and that is characterized the consequence of using different types of periodic texture. Our simulated results provide good agreement with updated amorphous silicon solar cell. The improvement of short-circuit current is enhanced by $36.16 \%$ than smooth substrate in textured cases. The short-circuit current density was obtained as $17.32 \mathrm{~mA} / \mathrm{cm}^{2}$ for the intrinsic layer thickness of $300 \mathrm{~nm}$. Contrastively, if we want to have an idea of conversion efficiency with reasonable open-circuit voltage of $900 \mathrm{mV}$ and $73 \%$ of fill-factor, [3] it is $11.38 \%$ as analyzed in our work.

\section{Acknowledgment}

The authors would like to thank Prof. Dietmar Knipp, Dr. Rahul Dewan, and Mr. Vladislav Jovanov for their all kinds of support to learn the subject matter during our master study. The lecture notes from the graduate course "Computational Electromagnetics" offered at Jacobs University Bremen, Germany by Prof. Jon Wallace was of great help for the formulation of the FDTD algorithm and understand the subject matter.

\section{References}

[1] B. Rech and H. Wagner, "Potential of Amorphous Silicon for Solar Cells," Applied Physics A: Material Science and Processing, vol. 69, Issue 2, pp. 155-167, 1999.

[2] J. Meier, J. Spitznagel, U. Kroll, C. Bucher, S. Faÿ, T. Moriarty, and A. Shah, "Potential of Amorphous and Microcrystalline Silicon Solar Cells," Thin Solid Films, vol. 451-452, pp. 518-524, 2004.

[3] Ping-Kuan Chang, Ting-Wei Kuo, Mau-Phon Houng, Chun-Hsiung Lu, Chih-Hung Yeh, "Effects of Temperature and Electrode Distance on Short-circuit Current in Amorphous Silicon Solar Cells, ”pp. 175-178, 18-25 May 2012.

[4] A. V. Shah, H. Schade, M. Vanecek, J. Meier, E. Vallat-Sauvain, N. Wyrsch, U. Kroll, C. Droz, and J. Bailat, "Thin-Film Silicon Solar Cell Technology," Progress in Photovoltaics: Research and Applications, vol. 12, Issue 2-3, pp. 113-142, March-May 2004.

[5] S. Fay", L. Feitknecht, R. Schlu" chter, U. Kroll, E. Vallat-Sauvain, A. Shah, "Rough ZnO Layers by LP-CVD Process and Their Effect in Improving Performances of Amorphous and Microcrystalline Silicon Solar Cells," Institut de Microtechnique (IMT), Rue A. - L. Breguet 2, vol. 90, no. 18-19, pp. 2960-2967, 2000 Neucha tel, Switzerland, Nov 2006. 
[6] J. Bailat, D. Dominé, R. Schlüchter, J. Steinhauser, S. Faÿ, F. Freitas, C. Bücher, L. Feitknecht, X. Niquille, T. Tscharner, A. Shah, C. Ballif, "High-Efficiency P-I-N Microcrystalline and Micromorph Thin Film Silicon Solar Cells deposited on LPCVD ZnO Coated Glass Substrates," Institut de Microtechnique, University of Neuchâtel, Breguet 2, CH-2000 Neuchâtel, Switzerland, 2006

[7] N. Bouchenak Khelladi, N. E. Chabane Sari, "Simulation Study of Optical Transmission Properties of $\mathrm{ZnO}$ Thin Film Deposited on Different Substrates", American Journal of Optics and Photonics. Vol. 1, no. 1, pp. 1-5, 2013.

[8] S. Lo, C. Chen, F. Garwe, and T. Pertch, "Broad-Band Anti-Reflection Coupler for a : Si Thin-Film Solar Sell," Journal of Physics D: Applied Physics, vol. 40, no. 3, pp. 754, 2007.

[9] C. Haase and H. Stiebig, "Thin-Film Silicon Solar Cells with Efficient Periodic Light Trapping Texture," Applied Physics Letter, vol. 91, pp. 061116, no. 6, 2007.
[10] J. Yang, A. Banerjee, and S. Guha, "Triple-junction amorphous silicon alloy solar cell with $14.6 \%$ initial and $13.0 \%$ stable conversion efficiencies," Applied Physics Letters, vol. 70, no. 22, pp. 2975-2977, 1997.

[11] K. Söderström, F.-J. Haug, J. Escarré, C. Pahud, R. Biron, and C. Ballif, "Highly reflective nanotextured sputtered silver back reflector for flexible high-efficiency n-i-p thin-film silicon solar cells," Solar Energy Materials and Solar Cells, vol. 95, no. 12, pp. 3585-3591, 2011.

[12] H. Sai, T. Koida, T. Matsui, I. Yoshida, K. Saito, and M. Kondo, "Microcrystalline silicon solar cells with $10.5 \%$ efficiency realized by improved photon absorption via periodic textures and highly transparent conductive oxide," Applied Physics Express, vol. 6, no. 10, Article ID 104101, 2013. 